\title{
Approximate Solutions of Two-Dimensional Burgers' and Coupled Burgers' Equations by Residual Power Series Method
}

\author{
Selahattin Gulsen ${ }^{1, *}$, Mustafa Inc ${ }^{1}$, and Harivan R. Nabi ${ }^{2}$ \\ ${ }^{1}$ Department of Mathematics, Firat University, Elazig, Turkey \\ ${ }^{2}$ Department of Road Construction, Duhok Polytechnic University, Duhok, Iraq
}

\begin{abstract}
In this study, two-dimensional Burgers' and coupled Burgers' equations are examined by the residual power series method. This method provides series solutions which are rapidly convergent and their components are easily calculable by Mathematica. When the solution is polynomial, the method gives the exact solution using Taylor series expansion. The results display that the method is more efficient, applicable and accuracy and the graphical consequences clearly present the reliability of the method.
\end{abstract}

\section{Introduction}

Initial value problems (IVPs) of differential equations (DEs) appear in a significant number of applications such as physical, biological and chemical processes [1-3]. Since solutions of IVPs cannot be obtained with analytical methods, numerical and approximate methods have been always constituted to get approximate solutions by some authors (see [4-6]). The previous studies do not give a methodical way to find solutions of IVPs and also previous studies require more time and more work to accomplish the results.

In this study, we apply the residual power series method (RPSM) to obtain approximate solutions of two-dimensional Burgers' and inhomogeneous coupled Burgers' equations. RPSM was firstly introduced by Arqub [7] to find series solutions of linear and nonlinear fuzzy problems in 2013. Arqub applied RPSM to the first-order and second-order fuzzy DEs and gave series solutions of these equations. Then Al-Smadi [8] carried out RPSM to linear and nonlinear IVPs in 2013. Alquran [9] fulfilled RPSM to nonlinear timefractional foam drainage equation to achieve approximate solution in 2014. El-Ajou [10] et al. found approximate solution of nonlinear fractional $\mathrm{KdV}$-Burgers equation with timespace-fractional derivatives with RPSM in 2015. Moaddy et al. [11] utilized RPSM to differential algebraic equations system in 2015. Tchier et al. [12] considered nonlinear time fractional reaction-diffusion equation with RPSM in 2016. Momani et al. [13] introduced RPSM for linear and nonlinear systems of IVPs and carried out on the effectiveness of RPSM in 2016. Mahmood and Yousif [14] applied RPSM to the coupled strong system nonlinear Boussinesq-Burgers equations to achieve approximate solutions

* Corresponding author: sgulsen2301@gmail.com 
and compared these solutions with other approximate solutions which found in other methods in 2017. Many scientists have applied RPSM to different equations and found approximate solutions of these equations (see [15-18]). RPSM is a semi analytical method which can be directly applied to IVPs to achieve series solution of them without linearization, perturbation and discretization [7]. In polynomial form, an analytical approximate solution is established by this method. RPSM and traditional higher order Taylor series method different from each other. As traditional Taylor series method is very expensive in high order computations, RPSM is very cheap and applicable to high orders. In practise this method can be called an alternative method to acquire analytic Maclaurin series solution of IVPs. Considering error concept for the method, we get a series solution, indeed this solution is a truncated series solution. The RPSM method consists of the following steps; firstly the method construct Maclaurin expansion of the solution, exact solution can be obtained when the solution is polynomial. Furthermore the solutions and all derivatives can be applied for each arbitrary point. Secondly, RPSM needs less time to obtain high precision solution with small computational requirements. In this article, we examine two-dimensional Burgers' and inhomogeneous coupled Burgers' equations. The Burgers equation has been found to explain the appromixate flow theory from a schock wave moving in a viscous fluid [19,20]. Fletcher [21] used the Hopf-Cole transformation to get an analytic solution of two-dimensional Burgers' equations in 1983. Because of the wide applicability of these equations, many scientists have applied different numerical methods to find approximate solutions of these equations (see [22-27]). This study consists of the following steps : In Section 2, we give the fundemental scheme of RPSM on twodimensional Burgers' equation. In Sections 3 and 4, RPSM is applied to two-dimensional Burgers' equation and inhomogeneous coupled Burgers' equation with appropriate initial conditions and obtained results are shown in graphs. Finally, consequences are given in section 5 .

\section{Analysis of Residual Power Series Method (RPSM) for Two- Dimensional Burgers' Equation}

To demonstrate the fundamental scheme of RPSM, we consider two-dimensional Burgers' equation

$$
\begin{aligned}
& u_{t}+u u_{x}+v u_{y}-\frac{1}{R e}\left(u_{x x}+u_{y y}\right)=0 \\
& v_{t}+u v_{x}+v v_{y}-\frac{1}{\operatorname{Re}}\left(v_{x x}+v_{y y}\right)=0
\end{aligned}
$$

with the initial conditions

$$
u(x, y, 0)=\frac{3}{4}-\frac{1}{4\left(1+\exp \left(\frac{\operatorname{Re}(-x+y)}{8}\right)\right)}, \quad v(x, y, 0)=\frac{3}{4}+\frac{1}{4\left(1+\exp \left(\frac{\operatorname{Re}(-x+y)}{8}\right)\right)},
$$

where $R e$ is the Reynolds number.

The solutions of the equations (1) and (2) depending on the conditions (3) are expressed as a series expansion around an initial point $t=0$ in the RPSM. Now, suppose that the solutions have the forms as

$$
u(x, y, t)=\sum_{m=0}^{\infty} f_{m}(x, y) t^{m}, \quad v(x, y, t)=\sum_{m=0}^{\infty} g_{m}(x, y) t^{m},
$$


where $m=0,1,2 \ldots$. Let us then define the expressions $u_{k}(x, y, t)$ and $v_{k}(x, y, t)$ as the $\mathrm{k}$ th truncated series of $u(x, y, t)$ and $v(x, y, t)$, respectively, i.e.

$$
u_{k}(x, y, t)=\sum_{m=0}^{k} f_{m}(x, y) t^{m}, \quad v_{k}(x, y, t)=\sum_{m=0}^{k} g_{m}(x, y) t^{m},
$$

where $k=1,2,3, \ldots$. Clearly, $u(x, y, t)$ and $v(x, y, t)$ provides the initial conditions (3), therefore the 0 -th RPSM approximate solutions of $u(x, y, t)$ and $v(x, y, t)$ are

$$
u(x, y, 0)=f_{0}(x, y)=f(x, y), v(x, y, 0)=g_{0}(x, y)=g(x, y) .
$$

Then, the estimated initial approach the first RPSM approximation solutions of $u(x, y, t)$ and $v(x, y, t)$ using equations (5) should be

$$
u_{1}(x, y, t)=f(x, y)+f_{1}(x, y) t, \quad v_{1}(x, y, t)=g(x, y)+g_{1}(x, y) t .
$$

So, expansion of equations (5) are reformulated as

$$
u_{k}(x, y, t)=f(x, y)+f_{1}(x, y) t+\sum_{m=2}^{k} f_{m}(x, y) t^{m}, \quad v_{k}(x, y, t)=g(x, y)+g_{1}(x, y) t+\sum_{m=2}^{k} g_{m}(x, y) t^{m},
$$

where $k=2,3, \ldots$. To find the values of coefficients $f_{m}(x, y)$ and $g_{m}(x, y), m=1,2,3, \ldots, k$ in series expansion of the equations (8), one can define the residual functions as

$$
\operatorname{Res}_{u}(x, y, t)=u_{t}+u u_{x}+v u_{y}-\frac{1}{\operatorname{Re}}\left(u_{x x}+u_{y y}\right), \quad \operatorname{Res}_{v}(x, y, t)=v_{t}+u v_{x}+v v_{y}-\frac{1}{\operatorname{Re}}\left(v_{x x}+v_{y y}\right)
$$

and so the $k$ - th residual functions can be expressed as

$$
\begin{aligned}
& \operatorname{Res}_{u, k}(x, y, t)=\left(u_{k}\right)_{t}+u_{k}\left(u_{k}\right)_{x}+v_{k}\left(u_{k}\right)_{y}-\frac{1}{\operatorname{Re}}\left(\left(u_{k}\right)_{x x}+\left(u_{k}\right)_{y y}\right), \\
& \operatorname{Res}_{v, k}(x, y, t)=\left(v_{k}\right)_{t}+u_{k}\left(v_{k}\right)_{x}+v_{k}\left(v_{k}\right)_{y}-\frac{1}{\operatorname{Re}}\left(\left(v_{k}\right)_{x x}+\left(v_{k}\right)_{y y}\right),
\end{aligned}
$$

where $k=1,2,3, \ldots$ Clearly, $\operatorname{Res}(x, y, t)=0, \lim _{k \rightarrow \infty} \operatorname{Res}_{k}(x, y, t)=\operatorname{Res}(x, y, t)$ for all $x \in I$ and $t \geq 0$. Therefore, when $t=0$ for each $s=0,1, \ldots, k, \partial^{s} \operatorname{Res}(x, y, t) / \partial t^{s}=0$. To obtain the coefficients $f_{m}(x, y)$ and $g_{m}(x, y), m=1,2,3, \ldots, k$, we carry out the following steps: Firstly, to employ the $m$-th truncated series of $u(x, y, t)$ and $v(x, y, t)$, we put on the derivative formula $\partial^{s} R \operatorname{es}(x, y, t) / \partial t^{s}=0$ in $\operatorname{Res}_{k}(x, y, t), m=1,2,3, \ldots, k$. In the obtained formula, we write $t=0$ and we equate it to zero. Lastly, to achieve the other coefficients, we solve the found algebraic formula

$$
\left(\partial^{s} R \text { e } s_{k}(x, y, t) / \partial t^{s}\right)=0(t=0), s=1,2,3, \ldots, k .
$$

Thus all coefficients of the multiple power series of equations (1)-(4) can be calculated.

\section{Application of Residual Power Series Method (RPSM) for Two-Dimensional Burgers' Equation}

The exact solutions of two-dimensional Burgers' problem (1)-(4) are 


$$
u(x, y, t)=\frac{3}{4}-\frac{1}{4\left(1+\exp \left(\frac{\operatorname{Re}(-t-4 x+4 y)}{32}\right)\right)}, \quad v(x, y, t)=\frac{3}{4}+\frac{1}{4\left(1+\exp \left(\frac{\operatorname{Re}(-t-4 x+4 y)}{32}\right)\right)} .
$$

With the iteration of RPSM for $n=0,1, \ldots$ it is obtained

$$
\begin{aligned}
& u_{1}(x, y, t)=\frac{3}{4}-\frac{1}{4\left(1+\exp \left(\frac{\operatorname{Re}(-x+y)}{8}\right)\right)}-\frac{\operatorname{Rt} \exp \left(\frac{\operatorname{Re}(-x+y)}{8}\right)}{128\left(1+\exp \left(\frac{\operatorname{Re}(-x+y)}{8}\right)\right)^{2}}, \\
& v_{1}(x, y, t)=\frac{3}{4}+\frac{1}{4\left(1+\exp \left(\frac{\operatorname{Re}(-x+y)}{8}\right)\right)}+\frac{\operatorname{Rt} \exp \left(\frac{\operatorname{Re}(-x+y)}{8}\right)}{128\left(1+\exp \left(\frac{\operatorname{Re}(-x+y)}{8}\right)\right)^{2}},
\end{aligned}
$$

and in this way $u_{2}(x, y, t), u_{3}(x, y, t), \ldots$, and $v_{2}(x, y, t), v_{3}(x, y, t), \ldots$, can be obtained and then the solutions are found as following

$$
u(x, y, t)=u_{1}(x, y, t)+u_{2}(x, y, t)+\ldots, \quad v(x, y, t)=v_{1}(x, y, t)+v_{2}(x, y, t)+\ldots .
$$
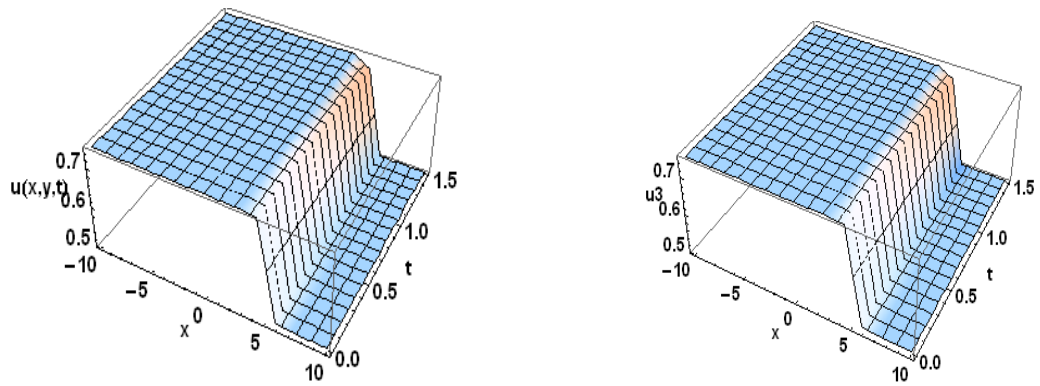

Figure 1. The surface graphs of the exact solution $u(x, y, t)$ and the approximate solution $u_{3}(x, y, t)$ of two-dimensional Burgers' problem when $y=5, R=50$, respectively.
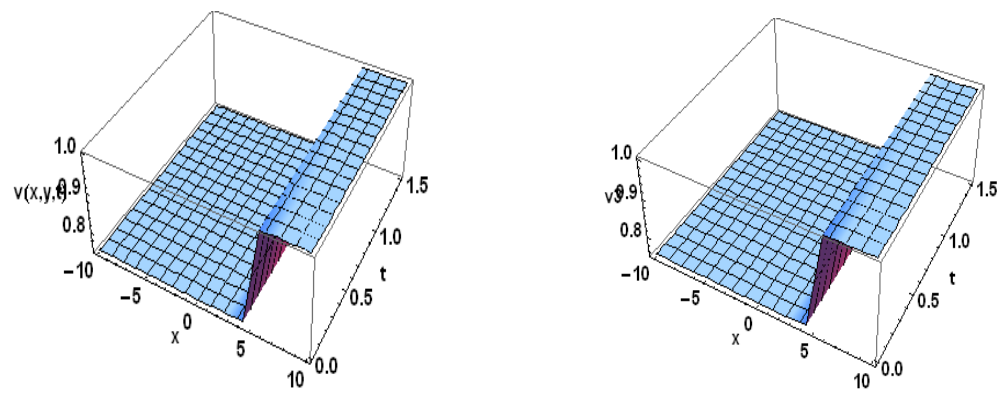

Figure 2. The surface graphs of the exact solution $v(x, y, t)$ and the approximate solution $v_{3}(x, y, t)$ of two-dimensional Burgers' problem when $y=5, R=50$, respectively. 


\section{Application of Residual Power Series Method (RPSM) for Inhomogeneous Coupled Burgers' Equation}

We consider inhomogeneous coupled Burgers' equation

with the initial conditions

$$
\begin{aligned}
& u_{t}-u_{x x}+2 u u_{x}+(u v)_{x}=F(x, t), \\
& v_{t}-v v_{x x}+2 v v_{x}+(u v)_{x}=G(x, t),
\end{aligned}
$$

where

$$
u(x, 0)=0, v(x, 0)=0
$$

The exact solutions of inhomogeneous coupled Burgers' problem (26)-(28) are

$$
u(x, t)=x^{2} t, \quad v(x, t)=\frac{t}{x} .
$$

With the iteration of RPSM for $n=0,1, \ldots$ it is found

and similarly

$$
u_{1}(x, t)=x^{2} t, \quad v_{1}(x, t)=\frac{t}{x},
$$

$$
u_{2}(x, t)=x^{2} t, \quad v_{2}(x, t)=\frac{t}{x}, \quad u_{3}(x, t)=x^{2} t-\frac{2 t^{3} x^{3}}{3}, \quad v_{3}(x, t)=\frac{t^{3}}{3 x^{3}}+\frac{t}{x},
$$

and in this way $u_{4}(x, t), u_{5}(x, t), \ldots$, and $v_{4}(x, t), v_{5}(x, t), \ldots$, can be obtained and then the solutions are found as following

$$
u(x, t)=u_{1}(x, t)+u_{2}(x, t)+\ldots ., \quad v(x, t)=v_{1}(x, t)+v_{2}(x, t)+\ldots .
$$
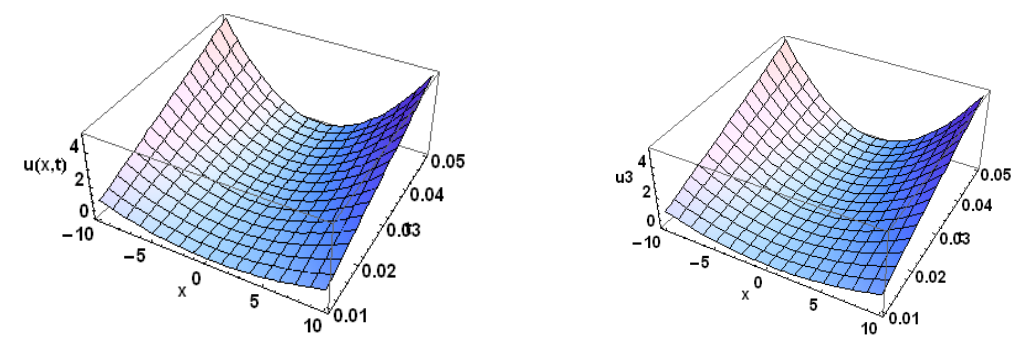

Figure 3. The surface graphs of the exact solution $u(x, t)$ and the approximate solution $u_{3}(x, t)$ of inhomogeneous coupled Burgers' problem, respectively.
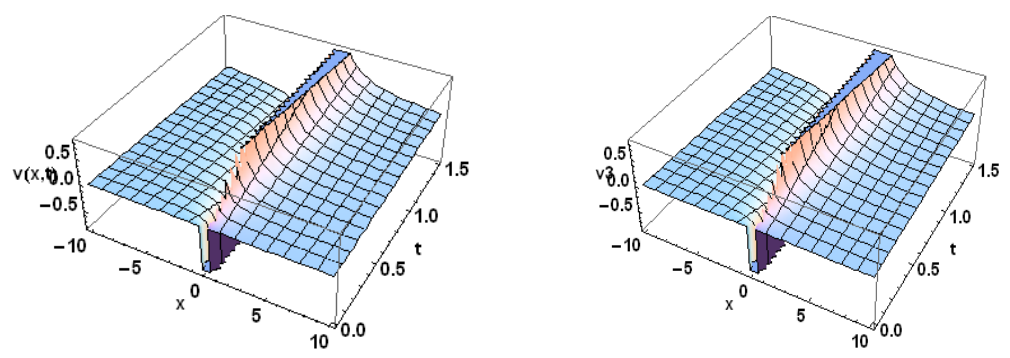

Figure 4. The surface graphs of the exact solution $v(x, t)$ and the approximate solution $v_{3}(x, t)$ of inhomogeneous coupled Burgers' problem, respectively. 


\section{Conclusion}

In this study, approximate solutions of the two-dimensional Burgers equation and inhomogeneous coupled Burgers equation were calculated by RPSM. It is seen that the results obtained with the RPSM are very close to the exact solutions. The obtained results were compared with the exact solutions and the obtained results are shown in graphs.

\section{References}

1. G. B. Whitham, John Wiley \& Sons, (1974)

2. M.W. Hirsch, S. Smale, Academic, New York, (1974)

3. L. Collatz, New York: John Wiley \& Sons, (1986)

4. J.R. Cash, A.H. Karp, ACM Trans. Math. Software (TOMS), 16, 201-222 (1990)

5. M.J. Jang, C.L. Chen, Y.C. Liy, Appl. Math. Comput., 115, 145-160. (2000)

6. I.A.H. Hassan, Chaos, Solitons \& Fractals, 36, 53-65 (2008)

7. O.A. Arqub, J. Adv. Res. Appl. Math., 5, 31-52 (2013)

8. M. Al-Smadi, Theoret. Math. Appl., 3, 199-210 (2013)

9. M. Alquran, Math. Sci., 8, 153-160 (2014)

10. A. El-Ajou, O.A. Arqub, S. Momani, J. Comput. Phys., 293, 81-95 (2015)

11. K. Moaddy, M. Al-Smadi, I. Hashim, Discrete Dyn. Nat. Soc., 2015, 12 pp. (2015)

12. F. Tchier, M. Inc, Z.S. Korpinar, D. Baleanu, Adv. Mech. Eng., 8, 1-10 (2016)

13. S. Momani, O.A. Arqub, M.A. Hammad, A. Alsaedi, Appl. Math. Inf. Sci, 10, 765-775 (2016)

14. B.A. Mahmood, M.A. Yousif, Cogent Math. Stat., 4, 11 pp. (2017)

15. I. Komashynska, M. Al-Smadi, A. Ateiwi, S. Al-Obaidy, Appl. Math, 10, 1-11 (2016)

16. A. Kumar, S. Kumar, M. Singh, Commun. Numer. Anal., 2016, 1-10 (2016)

17. L. Wang, X. Chen, Entropy, 17, 6519-6533 (2015)

18. M. Ali, I. Jaradat, M. Alquran, J. Math. Computer Sci., 17, 106-114 (2017)

19. J.D. Cole, Quart. Appl. Math., 9, 225-236 (1951)

20. J.M. Burger, Adv. Appl. Mech., 1, 171-199 (1948)

21. C.A. Fletcher, Int. J. Numer. Methods Fluids, 3, 213-216 (1983)

22. P.C. Jain, D.N. Holla, Internat. J. Non-Linear Mech., 13, 213-222 (1978)

23. A.R. Bahadir, Appl. Math. Comput., 137, 131-137 (2003)

24. S.M. El-Sayed, D. Kaya, Appl. Math. Comput., 158, 101-109 (2004)

25. M.A. Abdou, A.A. Soliman, J. Comput. Appl. Math., 181, 245-251 (2005)

26. A.K. Alomari, M.S.M. Noorani, R. Nazar, Appl. Math. Sci., 2, 1963-1977 (2008)

27. R. Abazari, A. Borhanifar, Comput. Math. Appl., 59, 2711-2722 (2010) 